Short Communication

\title{
Post-weaning social isolation of rats induces reduction in the gene expression of vascular endothelial growth factor (VEGF) in the hippocampus
}

\author{
Magdalena Chmelova $^{1}$, Daniela Jezova ${ }^{1}$, Igor Riecansky ${ }^{2,3}$ and Natasa Hlavacova ${ }^{1}$ \\ ${ }^{1}$ Department of Endocrine Regulations and Psychopharmacology, Institute of Experimental Endocrinology, Biomedical \\ Research Center, Slovak Academy of Sciences, Bratislava, Slovakia \\ 2 Department of Behavioural Neuroscience, Institute of Normal and Pathological Physiology, Centre of Experimental Medicine, \\ Slovak Academy of Sciences, Bratislava, Slovakia \\ ${ }^{3}$ Social, Cognitive and Affective Neuroscience Unit, Institute of Basic Psychological Research and Research Methods, Faculty \\ of Psychology, University of Vienna, Vienna, Austria
}

\begin{abstract}
The role of vascular endothelial growth factor (VEGF) in chronic stress and neurodevelopmental disorders is of growing research interest. Here we show that post-weaning isolation rearing of rats decreased gene expression of VEGF in the hippocampus. Gene expression of VEGF upstream regulator fibroblast growth factor-2 (FGF-2) or its downstream mediator endothelial nitric oxide synthase (eNOS) was unchanged. Other signaling pathways appear to be involved in isolationinduced reduction in VEGF gene expression. Sex differences in VEGF and eNOS gene expression with significantly higher mRNA levels in females than males were revealed.
\end{abstract}

Key words: Isolation - Sex differences - Growth factors - Gene expression - Hippocampus

Vascular endothelial growth factor (VEGF) is a potent growth factor playing a crucial role in vasculogenesis and angiogenesis. Intensive research over the last decade has brought evidence that VEGF exerts direct action in different neural cell types to produce neurotrophic and neuroprotective effects (Licht et al. 2011; Howell and Armstrong 2017). It has been suggested that the disruption in VEGF signaling during early life can impair neural development, and thus underlie the severe behavioral and cognitive deficits observed in neurodevelopmental disorders. Thus, the role of VEGF in neurodevelopmental pathologies is a subject of increasing research interest (Howell and Armstrong 2017).

The expression of VEGF is modulated by fibroblast growth factor-2 (FGF-2), a potent angiogenesis inducer (Cross and Claesson-Welsh 2001). VEGF is an important downstream mediator of the FGF-2 activity in the endothelial cells (Seghe-

Correspondence to: Natasa Hlavacova, Department of Endocrine Regulations and Psychopharmacology, Institute of Experimental Endocrinology, Biomedical Research Center, Slovak Academy of Sciences, Dubravska cesta 9, 845 05, Bratislava, Slovakia E-mail: natasa.hlavacova@savba.sk zzi et al. 1998). FGF-2 has been shown to stimulate proliferation and differentiation of neuroprogenitor cells in the adult hippocampus after brain insult (Yoshimura et al. 2001). On the other hand, one of the downstream mediators of VEGF action is endothelial nitric oxide synthase (eNOS) (Fukumura et al. 2001; Guo et al. 2016), which has been implicated in angiogenesis as well as in neurogenesis (Reif et al. 2004) and synaptic plasticity (Hopper and Garthwaite 2006).

There are several animal models allowing to approach mechanisms involved in neurodevelopmental pathological states. Isolation rearing of rats from weaning produces lasting developmental changes in behavior and brain neurobiology that may have translational pathophysiological relevance to alterations seen in schizophrenia or affective disorders (Fone and Porkess 2008). Due to the involvement of VEGF in neurodevelopmental processes, we have predicted that post-weaning social isolation of male and female rats results in decreased expression of VEGF in the hippocampus. Since this indeed turned out to be the case, in the second step we explored whether isolation rearing also affected the expression of FGF-2 and eNOS as an upstream regulator and downstream mediator of VEGF action, respectively. 
A total of 42 male and female Sprague-Dawley pups (AnLab, Prague, Czech Republic) were used in this study. At weaning, rats from both sexes were randomly assigned to the isolation-reared (one rat per cage) and socially-reared (3 rats of the same sex per cage) groups ( $n=9-12$ per sex and group). Animals were reared under these conditions from postnatal day 21 for 9 weeks. They were housed in plastic cages $(55.5 \times 34.5 \times 19.5 \mathrm{~cm}$ for social groups and $43.5 \times 28$ $\times 23 \mathrm{~cm}$ for isolation groups) containing sawdust bedding with metal grid lids, and had visual, auditory and olfactory contact with other rats in the same animal room. Animals were kept under standard housing conditions with a constant 12:12 h light/dark cycle (lights on at 06:00 h), temperature $\left(22^{\circ} \mathrm{C} \pm 2^{\circ} \mathrm{C}\right)$ and humidity $(55 \pm 10 \%)$. Food and water were available ad libitum. All experimental procedures were approved by the Animal Health and Animal Welfare Division of the State Veterinary and Food Administration of the Slovak Republic and conformed to the EU Directive 2010/63/EU on the protection of animals used for scientific purposes.

Following 9 weeks of isolation or social rearing, animals from all experimental groups were quickly decapitated with a guillotine. The brain was quickly removed from the skull. The hippocampus was quickly removed, frozen in liquid nitrogen and stored at $-70^{\circ} \mathrm{C}$ until analyzed. Real-time qPCR was used for quantitative evaluation of gene expression of VEGF, FGF-2 and eNOS in the hippocampus. Total RNA from the hippocampus was isolated and purificated by TRIzol ${ }^{\oplus}$ Reagent (Life Technologies, California, USA). Isolated RNA $(1 \mu \mathrm{g})$ was reverse-transcribed by oligo (dT) nucleotides using ProtoScript ${ }^{\circledast}$ First Strand cDNA Synthesis Kit (NEB, England). Real-time qPCR analysis was performed on a Fast Real-Time PCR System 7900 HT (Applied Biosystems, USA) using GoTaq qPCR Master Mix (Promega, USA) as described previously (Pokusa et al. 2016; Graban et al. 2017). Specific primers (Table 1) for VEGF, FGF-2 and eNOS were designed by Primer BLAST NCBI software. All data obtained by qPCR analysis were evaluated as an ng of mRNA (cDNA) according to a standard curve and was normalized to gene expression of peptidylprolyl isomerase A (PPIA) and TATA box binding protein (TBP) as reference genes (Table 1).
The obtained data were first checked for distributional properties by Shapiro Wilk's test and Levene's test and subsequently winsorized using a $15 \%$ two-tailed quantile trimming to treat the identified outlying observations $(1.5 \times$ interquartile range rule). All data were analyzed by two-way analysis of variance (ANOVA) with main factors of rearing conditions (social vs. isolation) and sex (male vs. female) followed by Tukey post-hoc test when appropriate. Partial $\eta^{2}$ was used as the measure of effect size in the ANOVA models. Pearson's correlation analysis was used to assess relationships among parameters studied. Results are expressed as means \pm SEM. The overall level of statistical significance was defined as $p<0.05$.

This study investigated the effects of post-weaning social isolation on gene expression of VEGF, FGF-2 and eNOS in the hippocampus of male and female rats. A two-way ANOVA revealed significant main effects of rearing conditions $\left(\mathrm{F}_{(1,38)}=11.19, p<0.01, \eta^{2}=0.23\right)$ and $\operatorname{sex}\left(\mathrm{F}_{(1,38)}=\right.$ $\left.7.43, p<0.05, \eta^{2}=0.16\right)$ on the gene expression of VEGF in the hippocampus (Fig. 1). Isolation rearing resulted in a reduction in mRNA levels coding for VEGF irrespective of sex, as indicated by a non-significant interaction between the factors. Generally, females exhibited higher VEGF mRNA levels than males. Neither rearing conditions nor sex significantly influenced the gene expression of FGF-2 in the hippocampus. There was no significant interaction between the rearing conditions and sex (Fig. 1). Statistical analysis did not reveal a significant effect of isolation rearing on the gene expression of eNOS (Fig. 1). There was, however, a significant effect of $\operatorname{sex}\left(\mathrm{F}_{(1,37)}=4.76, p<0.05, \eta^{2}=0.11\right)$ showing higher eNOS mRNA levels in females compared to males. No significant interaction between the factors was detected.

Pearson's correlation analysis revealed a moderate positive correlation between the hippocampal mRNA levels coding for VEGF and eNOS $(r=0.58, p<0.001)$ as well as VEGF and FGF-2 mRNA levels $(r=0.35, p<0.05)$. There was also a moderate positive correlation between eNOS and FGF-2 mRNA levels $(r=0.44, p<0.01)$

The present study showed that isolation rearing of rats from weaning led to a reduction in hippocampal gene expression of VEGF irrespective of sex. Somewhat surprisingly,

Table 1. Nucleotide sequence of primers used in gene expression evaluation of VEGF, FGF-2, eNOS, PPIA and TBP

\begin{tabular}{lll}
\hline Target gene & Forward primer $5^{\prime}-3^{\prime}$ & Reverse primer $5^{\prime}-\mathbf{3}^{\prime}$ \\
\hline VEGF & AAGACCGATTAACCATGTCA & ATGTCAGGCTTTCTGGATTA \\
FGF-2 & TCTATGGATCGCCTCACTTTGA & ACACATTGTATCCGTCCTTAAGCA \\
eNOS & GGGCTCCCTCCTTCCGGCTGC & GGATCCCTGGAAAAGGCG \\
PPIA & AAGCATACAGGTCCTGGCATCT & CATTCAGTCTTGGCAGTGCAG \\
TBP & TTCGTGCCAGAAATGCTGAA & GTTCGTGGCTCTCTTATTCTCATG \\
\hline
\end{tabular}

VEGF, vascular endothelial growth factor; FGF-2, fibroblast growth factor-2; eNOS, endothelial NOS; PPIA, peptidylprolyl isomerase A; TBT, TATA box binding protein. 

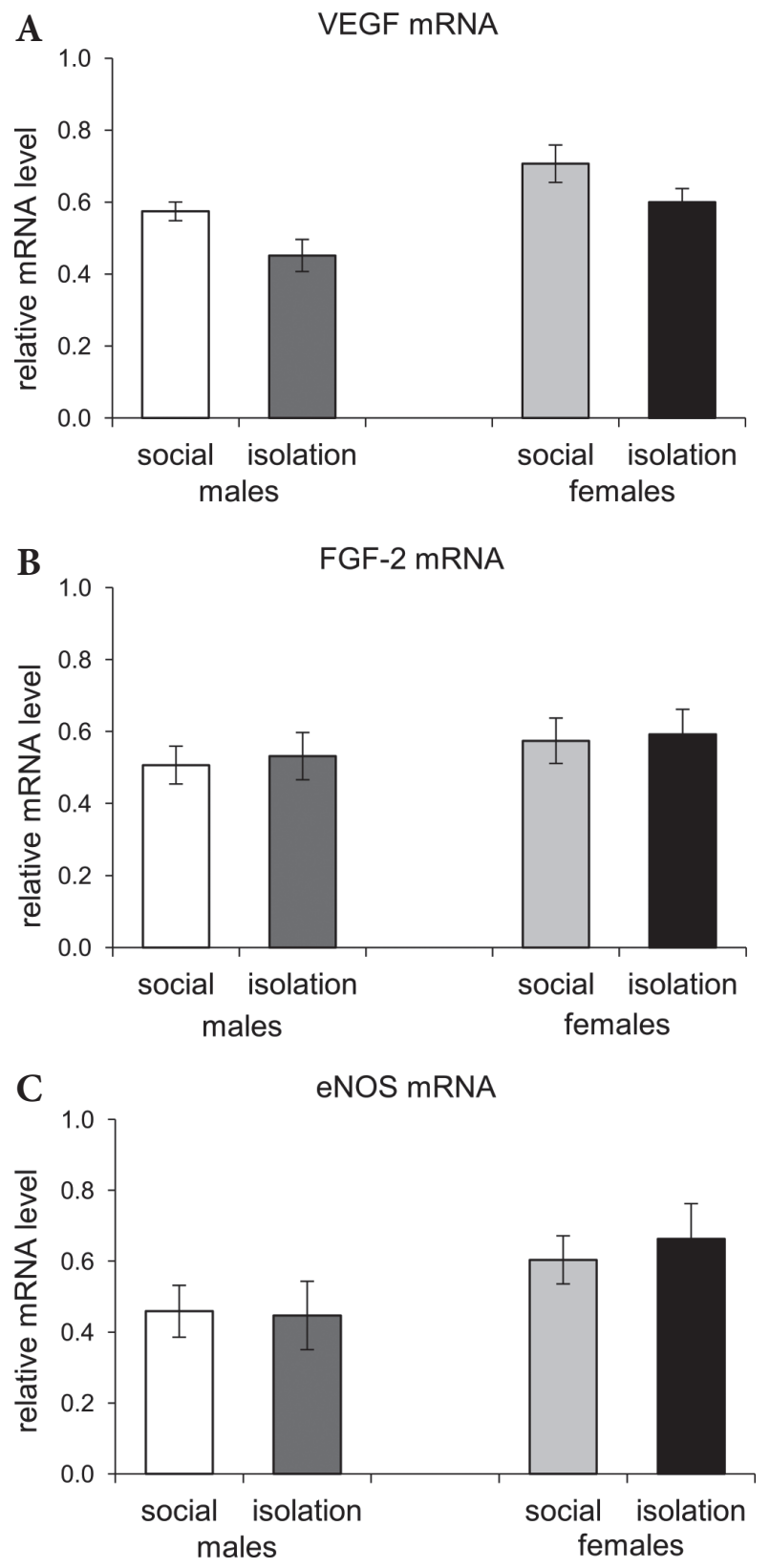

Figure 1. Gene expression of VEGF (A), FGF-2 (B) and eNOS (C) in the hippocampus of male and female rats reared in social isolation or in social groups from weaning for 9 weeks. Each value represents mean \pm SEM ( $n=12-15$ rats/group). Statistical significance as revealed by two-way ANOVA.

the gene expression of FGF-2 and eNOS, which are closely related to VEGF regulation and action, were not changed. Moreover, we revealed sex differences in VEGF and eNOS gene expression, with significantly higher mRNA levels in females compared to males.

This is the first study demonstrating the reduction in hippocampal VEGF mRNA levels in animals exposed to early adverse experience caused by post-weaning isolation rearing from weaning. Previous studies which reported a decreased expression of VEGF in the hippocampus of rats exposed to chronic stress or social isolation were performed on adult animals (Heine et al. 2005; Shilpa et al. 2017). Present results are in agreement with the utility of post-weaning social isolation of rats to induce brain alterations similar to those occurring in schizophrenia (Powell 2010; Murínová and Riečanský 2016; Murínová et al. 2017). In particular, a human post-mortem study has shown that VEGF mRNA levels are decreased in the brain tissue samples of schizophrenia patients (Fulzele and Pillai 2009). Relatedly, Storkebaum and Carmeliet (2004) proposed VEGF to play a role in the structural brain changes in schizophrenia.

Despite the finding of positive correlation between VEGF and eNOS as well as FGF-2 mRNA levels, the gene expression of these factors was not affected by isolation rearing. It is surprising since FGF-2 is an upstream regulator of VEGF expression and eNOS is a downstream effector of VEGF actions. It has been reported that both FGF-2 and VEGF increase nitric oxide production but only FGF-2 increases eNOS expression, indicating that these factors may differentially increase the activity and/or protein expression of eNOS in the endothelial cells (Mata-Greenwood et al. 2008). Present results strengthen this suggestion as the reduction in VEGF was not associated with changes in FGF-2 and eNOS.

An interesting finding of this study is the existence of sexual dimorphism in VEGF and eNOS gene expression in the hippocampus. Females showed higher mRNA levels coding for VEGF and eNOS than males. The authors of a recent study found no sex differences in VEGF mRNA copies in the hypothalamus, pituitary gland, and hippocampus (Obuchowicz et al. 2017). To our knowledge, sex difference in the eNOS expression level in the hippocampus has not yet been reported. It is tempting to speculate that the increased gene expression of VEGF and eNOS in females is due to estrogens, as $17 \beta$-estradiol was found to up-regulate VEGF expression in the hippocampus (Barouk et al. 2011).

The limitation of this study is that all measurements were performed only at the level of gene expression. The findings obtained should be verified at the protein level in future studies. In conclusion, post-weaning isolation rearing as a model of early stressful events impairing normal neurodevelopment in young rats reduces expression of VEGF in the hippocampus. Since parallel changes in FGF-2 and eNOS gene expression did not occur, other signaling pathways appear to be involved in isolation-induced reduction in VEGF expression. We brought the first evidence on sexual dimorphism in hippocampal VEGF and eNOS gene expression with higher levels in females. The present study supports the suggestion that VEGF is advancing towards being a novel biomarker and a potential therapeutic target in neurodevelopmental disorders. 
Acknowledgement. The authors are thankful to Jana Petova for her kind assistance. The study was supported by the Slovak Research and Development Agency (APVV, project number APVV-14-0840), and the Scientific Grant Agency of the Ministry of Education, Science, Research and Sports and the Slovak Academy of Sciences (project number VEGA 2/0042/19).

Conflict of interest. The authors declare that there are no conflicts of interest.

\section{References}

Barouk S, Hintz T, Li P, Duffy AM, MacLusky NJ, Scharfman HE (2011): $17 \beta$-estradiol increases astrocytic vascular endothelial growth factor (VEGF) in adult female rat hippocampus. Endocrinology 152, 1745-1751 https://doi.org/10.1210/en.2010-1290

Cross MJ, Claesson-Welsh L (2001): FGF and VEGF function in angiogenesis: signalling pathways, biological responses and therapeutic inhibition. Trends Pharmacol. Sci. 22, 201-207 https://doi.org/10.1016/S0165-6147(00)01676-X

Fukumura D, Gohongi T, Kadambi A, Izumi Y, Ang J, Yun CO, Buerk DG, Huang PL, Jain RK (2001): Predominant role of endothelial nitric oxide synthase in vascular endothelial growth factor-induced angiogenesis and vascular permeability. Proc. Natl. Acad. Sci. USA 98, 2604-2609 https://doi.org/10.1073/pnas.041359198

Fone KC, Porkess MV (2008): Behavioural and neurochemical effects of post-weaning social isolation in rodents-relevance to developmental neuropsychiatric disorders. Neurosci. Biobehav. Rev. 32, 1087-10102 https://doi.org/10.1016/j.neubiorev.2008.03.003

Fulzele S, Pillai A (2009): Decreased VEGF mRNA expression in the dorsolateral prefrontal cortex of schizophrenia subjects. Schizophr. Res. 115, 372-373 https://doi.org/10.1016/j.schres.2009.06.005

Graban J, Hlavacova N, Jezova D (2017): Increased gene expression of selected vesicular and glial glutamate transporters in the frontal cortex in rats exposed to voluntary wheel running. J. Physiol. Pharmacol. 68, 709-714

Guo H, Zhou H, Lu J, Qu Y, Yu D, Tong Y (2016): Vascular endothelial growth factor: an attractive target in the treatment of hypoxic/ischemic brain injury. Neural Regen. Res. 11, 174-179 https://doi.org/10.4103/1673-5374.175067

Heine VM, Zareno J, Maslam S, Joëls M, Lucassen PJ (2005): Chronic stress in the adult dentate gyrus reduces cell proliferation near the vasculature and VEGF and Flk-1 protein expression. Eur. J. Neurosci. 21, 1304-1314 https://doi.org/10.1111/j.1460-9568.2005.03951.x

Hopper RA, Garthwaite J (2006): Tonic and phasic nitric oxide signals in hippocampal long-term potentiation. J. Neurosci. 26, $11513-11521$ https://doi.org/10.1523/JNEUROSCI.2259-06.2006

Howell KR, Armstrong J (2017): Vascular endothelial growth factor (VEGF) in neurodevelopmental disorders. Curr. Behav. Neurosci. Rep. 4, 299-308 https://doi.org/10.1007/s40473-017-0130-9
Licht T, Goshen I, Avital A, Kreisel T, Zubedat S, Eavri R, Segal M, Yirmiya R, Keshet E (2011): Reversible modulations of neuronal plasticity by VEGF. Proc. Natl. Acad. Sci. USA 108, 5081-5086 https://doi.org/10.1073/pnas.1007640108

Mata-Greenwood E, Liao WX, Zheng J, Chen DB (2008): Differential activation of multiple signalling pathways dictates eNOS upregulation by FGF2 but not VEGF in placental artery endothelial cells. Placenta 29, 708-717 https://doi.org/10.1016/j.placenta.2008.05.005

Murínová J, Riečanský I (2016): Neurodevelopmental rat models of schizophrenia. Act. Nerv. Super. Rediviva 58, 43-46

Murínová J, Hlaváčová N, Chmelová M, Riečanský I (2017): The evidence for altered BDNF expression in the brain of rats reared or housed in social isolation: a systematic review. Front. Behav. Neurosci. 11, 101 https://doi.org/10.3389/fnbeh.2017.00101

Obuchowicz E, Nowacka M, Paul-Samojedny M, Bielecka-Wajdman AM, Małecki A (2017): Sex differences in the effect of acute peripheral IL- $1 \beta$ administration on the brain and serum BDNF and VEGF expression in rats. Cytokine 90, 6-13 https://doi.org/10.1016/j.cyto.2016.10.001

Pokusa M, Hlavacova N, Csanova A, Franklin M, Zorad S, Jezova D (2016): Adipogenesis and aldosterone: a study in lean tryptophan-depleted rats. Gen. Physiol. Biophys. 35, 379-386 https://doi.org/10.4149/gpb_2016020

Powell SB (2010): Models of neurodevelopmental abnormalities in schizophrenia. Curr. Top Behav. Neurosci. 4, 435-481 https://doi.org/10.1007/7854_2010_57

Reif A, Schmitt A, Fritzen S, Chourbaji S, Bartsch C, Urani A, Wycislo M, Mössner R, Sommer C, Gass P, Lesch KP (2004): Differential effect of endothelial nitric oxide synthase (NOSIII) on the regulation of adult neurogenesis and behaviour. Eur. J. Neurosci. 20, 885-895 https://doi.org/10.1111/j.1460-9568.2004.03559.x

Seghezzi G, Patel S, Ren CJ, Gualandris A, Pintucci G, Robbins ES, Shapiro RL, Galloway AC, Rifkin DB, Mignatti P (1998): Fibroblast growth factor-2 (FGF-2) induces vascular endothelial growth factor (VEGF) expression in the endothelial cells of forming capillaries: an autocrine mechanism contributing to angiogenesis. J. Cell Biol. 141, 1659-1673 https://doi.org/10.1083/jcb.141.7.1659

Shilpa BM, Bhagya V, Harish G, Srinivas Bharath MM, Shankaranarayana Rao BS (2017): Environmental enrichment ameliorates chronic immobilisation stress-induced spatial learning deficits and restores the expression of BDNF, VEGF, GFAP and glucocorticoid receptors. Prog. Neuropsychopharmacol. Biol. Psychiatry 76, 88-100 https://doi.org/10.1016/j.pnpbp.2017.02.025

Storkebaum E, Carmeliet P (2004): VEGF: a critical player in neurodegeneration. J. Clin. Invest. 113, 14-18 https://doi.org/10.1172/JCI20682

Yoshimura S, Takagi Y, Harada J, Teramoto T, Thomas SS, Waeber C, Bakowska JC, Breakefield XO, Moskowitz MA (2001): FGF-2 regulation of neurogenesis in adult hippocampus after brain injury. Proc. Natl. Acad. Sci. USA 98, 5874-5879 https://doi.org/10.1073/pnas.101034998

Received: April 15, 2019

Final version accepted: May 13, 2019

First published online: June 20, 2019 